\title{
Functional magnetic resonance imaging in primary hyperparathyroidism
}

\author{
Yunglin Gazes ${ }^{1}$, Minghao Liu², Melissa Sum ${ }^{3}$, Elaine Cong ${ }^{4}$, Jennifer Kuo ${ }^{5}$, James A Lee ${ }^{5}$, Shonni Silverberg ${ }^{4}$, \\ Yaakov Stern ${ }^{1}$ and Marcella Walker ${ }^{4}$
}

1'Department of Neurology, Columbia University Irving Medical Center, New York, New York, USA, ${ }^{2}$ Division of Endocrinology, Department of Medicine, Hofstra Northwell School of Medicine at Hofstra University, Hempstead, New York, USA, ${ }^{3}$ Division of Endocrinology, Department of Medicine, New York University Langone Medical Center, New York, New York, USA, ${ }^{4}$ Division of Endocrinology, Department of Medicine, Columbia University Irving Medical Center, New York, New York, USA, and ${ }^{5}$ Department of Surgery, Columbia University Irving Medical Center, New York, New York, USA

Correspondence should be addressed to M Walker

Email

mad2037@columbia.edu

\begin{abstract}
Objective: The neurophysiological mechanisms underlying cognitive dysfunction in primary hyperparathyroidism (PHPT) and the brain regions affected are not clear. We assessed neural activation during cognitive testing (matrix reasoning, paired associates, and logical memory) using functional MRI (fMRI) in 23 patients with PHPT and 23 healthy controls. A subset with PHPT was re-assessed 6 months post-parathyroidectomy (PTX).

Design: This is an observational study comparing neural activation by fMRI in patients with PHPT to normative controls. Postmenopausal women were studied at a tertiary referral center.

Results: There were no between-group differences in cognitive task performance. Patients with PHPT had lower neural activation vs controls ( $\max Z=4.02$, all $P<0.01$ ) during matrix reasoning in brain regions involved in executive function (left frontal lobe $(k=57)$ and right medial frontal gyrus $(k=72))$ and motor function (right precentral gyrus $(k=51)$ ). During paired associates (verbal memory), those with PHPT had greater activation in the right inferior parietal lobule (language/mathematical operations; $\mathrm{k}=65, P<0.01$ ). Greater activation in this region bilaterally correlated with higher PTH $(k=96, P<0.01)$. Post-PTX, activation decreased during matrix reasoning, but in different regions than those affected pre-PTX.

Conclusions: PHPT is associated with differences in task-related neural activation patterns, but no difference in cognitive performance. While this may indicate compensation to maintain the same cognitive function, there was no clear improvement in neural activation after PTX. Larger, longitudinal studies that include PHPT patients followed without surgery are needed to determine if PTX could prevent worsening of altered neural activation patterns in PHPT.
\end{abstract}

\section{Introduction}

PHPT is a common endocrine condition associated with hypercalcemia and elevated or inappropriately normal serum levels of parathyroid hormone (PTH). Patients with PHPT commonly report neuropsychological symptoms (1, $2,3,4)$. Many studies suggest that PHPT is associated with reduced quality of life (QOL), depression, and cognitive changes such as impaired memory, attention, or executive function $(5,6,7,8,9,10,11,12,13,14,15,16,17)$. Whether neuropsychological symptoms are specifically attributable to PHPT and reversible after parathyroidectomy (PTX) is controversial, and results from both observational studies and randomized trials have been conflicting $(5,8,9,10$, 18). Our prior work in patients with PHPT indicated worse performance on tests of verbal memory and non-verbal abstraction (executive function and pattern recognition) (5). Performance on these tasks improved after PTX (5).

Published by Bioscientifica Ltd. 
Although both hypercalcemia and high PTH have variably been associated with cognition in some, but not all, prior studies, the neurophysiological mechanism(s) underlying cognitive dysfunction and brain regions affected in PHPT have not been clearly elucidated and have rarely been investigated $(5,6,7,8,9,10,11,12,13,14,15,16,17)$.

Functional MRI, or fMRI, is a non-invasive tool that maps brain function. Functional MRI is based on the principle that cerebral blood flow and neuron activity (or neuronal activation) are coupled. When neurons are activated in response to a cognitive test or task, blood flow to that brain area increases. The increase in blood flow is followed by a relative increase in blood oxygenation (oxyhemoglobin) and a decrease in deoxyhemoglobin. Because deoxyhemoglobin and oxyhemoglobin vary in their magnetic properties, the change in blood oxygenation is detected as an increase in magnetic resonance signal that is able to 'map' the task to the brain region responsible.

In the clinical setting, fMRI has been used for pre-surgical mapping in patients with brain tumors and epilepsy. Functional MRI has also been useful in revealing mechanisms in Alzheimer's disease, depression, schizophrenia, lupus, cognitive aging, and other neurological and psychiatric disorders $(19,20)$. Only two studies ( $n=6$ and $n=18$ ) have applied fMRI to the study of cognitive dysfunction in PHPT $(13,21)$. Both were small and did not compare patients with PHPT to non-PHPT controls. In order to elucidate the neurophysiological mechanisms of cognitive dysfunction in PHPT, brain regions affected and the utility of functional imaging in this disorder, we assessed neural activation patterns using fMRI during verbal memory and executive function/ reasoning tasks, cognitive functions previously shown to be impaired in PHPT (5). fMRI was performed in patients with PHPT compared to controls derived from a normative fMRI database at baseline and after PTX in the subset who underwent surgery.

\section{Subjects and methods}

This study was approved by the Columbia University Medical Center (CUMC) Institutional Review Board and all participants provided written informed consent. Participants were recruited from the Metabolic Bone Diseases Unit and the endocrine surgery and general endocrinology clinics at CUMC between December 2013 and September 2017. Consecutive female postmenopausal patients with PHPT who met inclusion/exclusion criteria and agreed to participate were enrolled. Two-hundred fifty-three individuals with primary hyperparathyroidism were screened for enrollment. We included women with PHPT regardless of whether they planned to have PTX. PHPT $(n=23)$ was defined by the presence of hypercalcemia (calcium above normal $(>10.2 \mathrm{mg} / \mathrm{dL})$ with an elevated PTH level ( $>65 \mathrm{pg} / \mathrm{mL}$; normal range 10-65 pg/mL)). Normative sex-, age- and education-matched controls without PHPT $(n=23)$ were selected from a CUMC study that established 'normal' fMRI neural activation patterns for core cognitive abilities/tasks (episodic memory, fluid reasoning, etc.) and assessed changes in neural activation across the lifespan $(19,22,23)$. The same fMRI cognitive tasks and testing conditions were utilized for cases and controls (19). Controls were recruited between 2013 and 2017.

Postmenopausal women who were native English speakers between 50 and 85 years of age were eligible to participate. We excluded those who were left-handed because of known differences in neural localization of language functions based on handedness and those with conditions affecting cognition. Information on comorbidities and medications was collected by questionnaire as previously described (24). Both cases and controls were free of significant cardiovascular (active coronary artery disease or heart failure) or neurologic conditions (brain injury, tumor, infection, epilepsy, dementia, learning disability, uncontrolled major depression, mental retardation, multiple sclerosis, degenerative diseases, Parkinson's disease, Huntington's disease, and attention deficit disorder). We also excluded those with renal or liver disease, active malignancy other than non-melanomatous skin cancer, HIV/AIDS, alcohol/drug abuse, uncontrolled hyper/hypothyroidism, uncontrolled hypertension or diabetes, uncorrectable vision/hearing, or those who had MRI contraindications. Controls had no known history of parathyroid disease. We also excluded men and premenopausal women to avoid the confounding effects of sex or menopause status.

\section{Cognitive fMRI tasks}

All participants were studied at baseline. The subset of patients with PHPT who elected to have PTX returned 6 months after PTX. Tasks were selected based on our prior study that had indicated worse performance on tests of verbal memory and non-verbal abstraction (5). Prior to the scan session, computerized training was administered, and at the completion of training for each task, participants had the option of repeating the training. Responses were 
differential button presses. During training, responses were made on the computer keyboard and during scans they were made on the LUMItouch response system. The following tasks were administered: (1.) Logical Memory: Stories are presented on the computer screen. The subjects are asked to answer detailed multiple-choice questions about the story, with four possible answer choices; (2.) Paired Associates: Pairs of words are presented, one at a time, on the screen, and subjects are instructed to remember the pairs. Following the pairs, they were given a probe word at the top of the screen and four additional word choices below. Subjects were asked to choose the word that was originally paired with the probe word; and (3.) Matrix Reasoning: (adapted from Raven, 1962) Subjects are given a matrix that is divided into nine cells, in which the figure in the bottom right cell is missing. Below the matrix, they are given eight figure choices, and they are instructed to evaluate which of the figures would best complete the missing cell.

Task stimuli were back-projected onto a screen located at the foot of the MRI bed using an LCD projector. Participants viewed the screen via a mirror system located in the head coil and, if needed, had vision corrected to normal using MR compatible glasses (manufactured by SafeVision, LLC. Webster Groves, MO). Responses were made on a LUMItouch response system (Photon Control Company). Task administration and collection of reaction time (RT) and accuracy data were controlled by EPrime (v2.08) running on a PC computer. Task onset was electronically synchronized with the MRI acquisition computer. Task accuracy and reaction time were recorded.

\section{MR image acquisition and processing}

All MR images were acquired on a 3.0 T Philips Achieva Magnet and used a $240 \mathrm{~mm}$ field of view. For the EPI acquisition, the parameters were: TE/TR (ms) 20/2000; Flip Angle 72 degrees; In-plane resolution (voxels) $112 \times 112$; Slice thickness/gap $(\mathrm{mm}) 3 / 0 ; 41$ slices. In addition, T1 MPRAGE was acquired to obtain a structural image of the brain necessary for registration to standard template. Each individual's three fMRI scans were preprocessed in the same manner using the fMRIB Software Library (FSL; Smith et al. 2004). The processing of the functional imaging data involved the following basic steps: (1.) withinsubject histogram computation for each subject volume to identify noise (FEAT); (2.) subject-motion correction (MCFLIRT); (3.) slice-timing correction; (4.) brain-mask creation from first volume in subject's fMRI data; (5.) highpass filtering ( $\mathrm{T}=128 \mathrm{~s}$ ); (6.) pre-whitening; (7.) General-
Linear-Model (GLM) estimation with equally temporally filtered regressors and double-gamma hemodynamic response functions; and (8.) registration of functional and structural images with subsequent normalization into MNI space (FNIRT). GLM for each subject and each task consisted of block-based time series analysis from which contrast images were used in the group-level analyses. The analysis compares activation within each image voxel in subjects vs controls or before vs after PTX. ' $\mathrm{k}$ ' indicates the voxel cluster size. By convention, only clusters $>50$ contiguous voxels with $P<0.01$ are reported. Z-stat is the $z$-statistic from a standard transformation from the group level $t$-value of the statistical test used to determine whether the means differ between two groups.

\section{Biochemistries}

Fasting samples for serum calcium (normal 8.6-10.2 $\mathrm{mg} / \mathrm{dL})$, creatinine $(0.5-0.9 \mathrm{mg} / \mathrm{dL})$, and phosphate $(2.5-4.5$ $\mathrm{mg} / \mathrm{dL}$ ) were measured on a Cobas Integra 400 plus (Roche Diagnostics, Indianapolis, IN) with intra- and inter-assay precision $<3.0 \%$. Total intact PTH (normal $10-65 \mathrm{pg} / \mathrm{mL}$ ) was measured with an IRMA (Scantibodies Laboratories, Hanover, Germany) with intra- and inter-assay precision of $4.0 \%$ and $5.8 \%$, respectively. 25-hydroxyvitamin D2 and 25-hydroxyvitamin D3 were measured using Ultra Performance Liquid Chromatography-Tandem Mass Spectrometry (LC-MSMS). Inter-day precision was $8.1 \%$ for 25-OH-D2 and 5.5\% for 25-OH-D3.

\section{Statistical analysis}

Descriptive statistics were expressed as absolute $(n)$ and relative (\%) frequency and mean and S.D. for categorical and continuous demographics and biochemistries, respectively. Student's $t$-test, Chi-squared test or Fisher's exact test were used, as appropriate, to assess between-group differences in demographics and clinical characteristics. Paired t-tests were used to assess withinperson changes in biochemistries. A two-tailed $P$-value of $<0.05$ was considered statistically significant. SAS version 9.4 was used for analysis.

\section{Results}

\section{Clinical characteristics}

Those with PHPT had biochemically mild disease (mean \pm s.D.: serum calcium $10.4 \pm 0.3 \mathrm{mg} / \mathrm{dL}$, PTH 
83.2 $\pm 38.5 \mathrm{pg} / \mathrm{mL}, \quad 25$-hydroxyvitamin $\quad \mathrm{D} \quad 37.4 \pm 16.1$ $\mathrm{ng} / \mathrm{mL}$ ). In the PHPT group, $27.3 \%$ had a history of nephrolithiasis, $27.3 \%$ had osteoporosis, $18.2 \%$ had a history of fragility fracture, and $68.2 \%$ met one or more surgical criteria for PTX based on the 2014 Guidelines for the Management of Asymptomatic PHPT (25). Cases and controls did not differ in age or education or the frequency of comorbidities (Table 1). Participants with PHPT ranged between 53 and 77 years of age.

\section{Baseline assessment}

As shown in Fig. 1A and Table 2, patients with PHPT had reduced neural activation compared to controls during matrix reasoning in brain regions involved in executive function (the left frontal lobe $(\mathrm{k}=57$, Z-stat $=4.02, P<0.01)$ and right medial frontal gyrus $(\mathrm{k}=72, \mathrm{Z}$-stat $=3.24$, $P<0.01)$ and motor function (right precentral gyrus $(\mathrm{k}=51$, Z-stat $=3.08, P<0.01)$ ). Additionally, neural activation was lower in the left brainstem $(\mathrm{k}=90$, Z-stat $=3.64, P<0.01)$.

Among those with PHPT, we assessed the association between neural activation and the biochemical hallmarks of PHPT severity (Table 3). Higher activation was associated with higher PTH in an area associated with working memory (left superior frontal gyrus $(\mathrm{k}=55$, Z-stat $=3.33$, $P<0.01)$ ) and higher calcium during this task (left middle temporal gyrus $(\mathrm{k}=69$, Z-stat $=3.74 P<0.01)$ ). There was also a negative association between PTH and activation in the left caudate head (Table 3) during this task.

During paired associates (verbal memory), those with PHPT had greater activation than controls in the right inferior parietal lobule $(\mathrm{k}=65$, Z-stat $=3.71, P<0.01)$, an area involved in language and mathematical operations (Fig. 1B and Table 2). Greater activation in this region correlated with higher PTH $(\mathrm{k}=96$, Z-stat $=3.27, P<0.01)$ during this task (Table 3 ). Higher calcium also correlated with greater activation in the left middle temporal gyrus during this task $(\mathrm{k}=58, \mathrm{Z}$-stat $=3.71, P<0.01)$. There was no between-group difference in activation during the other verbal memory task, logical memory. As shown in Table 3, there was also a positive correlation between serum PTH and higher activation in the left middle frontal gyrus during logical memory (Table 3). There were no differences in accuracy or reaction time between those with and without PHPT on any of the three tasks (data not shown).

\section{Longitudinal assessment}

Post-PTX fMRI was available on 15 participants, but one subject did not have data on matrix reasoning and one did not have paired associates data (i.e. image files corrupted). Those who participated in the longitudinal follow-up $(n=15)$ did not differ from those who did not $(n=8)$ by age $(63.9 \pm 5.9$ vs $61.9 \pm 5.8, P=0.43)$, education $(17.6 \pm 1.9$ vs $18.0 \pm 1.4, P=0.61)$, serum calcium $(10.4 \pm 0.4$ vs $10.5 \pm 0.4, P=0.85)$, or PTH $(77.2 \pm 30.5$ vs $96.1 \pm 52.2$, $P=0.29)$. All participants were cured by surgery. As expected, after PTX, serum calcium and PTH normalized and 25-hydroxyvitamin D and serum phosphate increased in the PHPT group, while renal function did not change (Table 4). As shown in Fig. 2, after PTX, neural activation decreased during matrix reasoning, but in different brain regions than were affected pre-PTX: the largest cluster consisted of bilateral precuneus, angular gyri and cuneus, and the left lingual gyrus $(\mathrm{k}=961$, Z-stat $=4.04$, $P<0.01)$ and the second cluster was part of the left posterior cingulate gyrus $(\mathrm{k}=52, \mathrm{Z}$-stat $=3.12, P<0.01)$, which are involved in complex visual processing and attention, respectively. Activation also decreased during the logical memory task after PTX in the left precuneus (cluster 1: $\mathrm{k}=161, \mathrm{Z}$-stat $=3.48, P<0.01$; cluster 2 : $\mathrm{k}=120$, Z-stat $=3.62, P<0.01)$ and middle occipital gyrus $(\mathrm{k}=120$, Z-stat $=3.36, P<.01)$, though activation was not different

Table 1 Demographics and co-morbidities.

\begin{tabular}{|c|c|c|c|}
\hline & PHPT & Controls & P-value \\
\hline Age (years) & $63.2 \pm 5.8$ & $63.3 \pm 5.8$ & 0.96 \\
\hline Education (years) & $17.7 \pm 1.8$ & $17.3 \pm 1.1$ & 0.33 \\
\hline $\mathrm{BMI}\left(\mathrm{kg} / \mathrm{m}^{2}\right)$ & $26.7 \pm 3.9$ & $25.0 \pm 4.1$ & 0.20 \\
\hline Diabetes (\%) & $4.8 \%$ & $5.0 \%$ & 1.0 \\
\hline Hypertension (\%) & $22.7 \%$ & $19.5 \%$ & 1.0 \\
\hline Peripheral vascular disease (\%) & $0 \%$ & $0 \%$ & 1.0 \\
\hline Current smoking (\%) & $0 \%$ & $5.0 \%$ & 0.49 \\
\hline Anti-hypertensive use (\%) & $17.4 \%$ & $14.3 \%$ & 0.70 \\
\hline Cholesterol-lowering medication (\%) & $22.7 \%$ & $9.5 \%$ & 0.41 \\
\hline
\end{tabular}




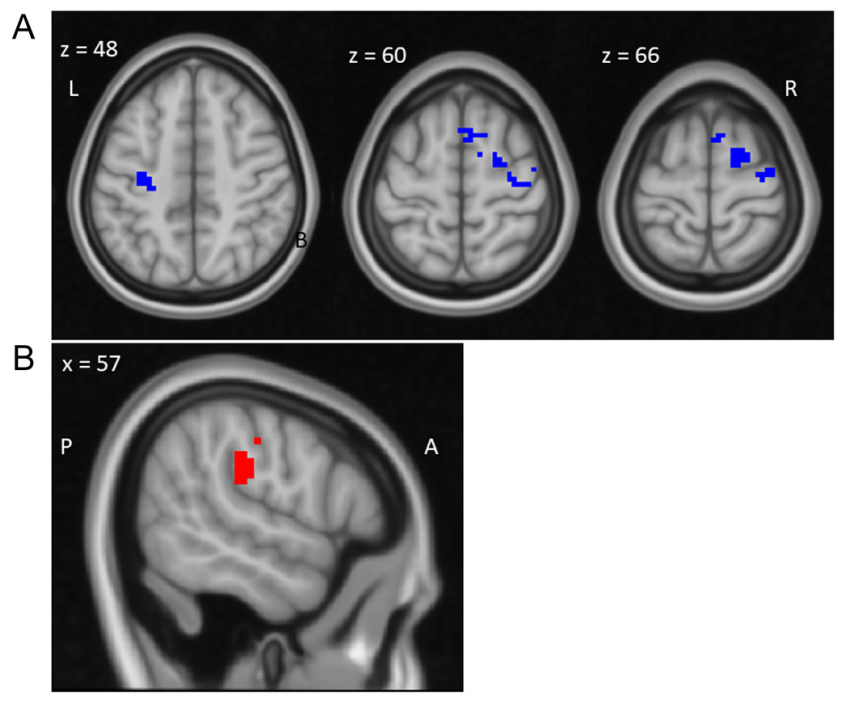

Figure 1

Representative $\mathrm{fMRI}$ images indicating neural activation in PHPT compared to controls during $(A)$ matrix reasoning and (B) paired associates tasks. In A, activation is lower (blue) in patients with PHPT compared to healthy controls in the regions shown. During paired associates (B), those with PHPT had greater (red) activation than controls. $Z$ refers to the Montreal Neurological Institute (MNI) coordinates in the inferior-superior spatial plane. $X$ refers to the $\mathrm{MNI}$ coordinates in the left-right plane; $L=$ left; $R$ = right; $A$ = anterior; $\mathrm{P}=$ posterior.

between PHPT and controls during logical memory before PTX. Reaction time decreased after PTX on all tasks (data not shown), consistent with a learning effect.

\section{Discussion}

In this study, we assessed neural activation patterns in patients with PHPT compared to normal reference patterns among controls in order to assess neurophysiological mechanisms that may underlie cognitive changes in this condition as well as brain regions affected. To our knowledge, this is the largest study to utilize fMRI in PHPT and the only study to compare neural activation in PHPT to controls without PHPT. A major strength of our study is that our age- and education-matched controls were derived from a normal reference population database that established standard fMRI activation patterns for the given tasks (22). Our results indicate that task-related neural activation differs between those with and without PHPT in several regions of the brain involved in essential processing of the task demands, including executive function, attention, visual processing, and motor control. The areas in which the differences were localized are consistent with the expected brain regions and networks of brain activity for these tasks based on activation patterns in healthy adults (23). Further, we found an association between the biochemical hallmarks of PHPT severity and activation in some of the brain regions where neural activation was higher (parietal lobe during paired associates) in cases pre-operatively compared to controls.

While PHPT was associated with differences in the level of brain activity, task performance was similar. This may suggest that altered neural processing is needed in PHPT to maintain similar cognitive function compared to controls. The direction of the differences (higher or lower activation compared to controls) varied by task. We found greater activation in PHPT vs controls during the paired associates (verbal memory) task in the inferior parietal lobule, but lower activation during matrix (fluid) reasoning in other brain regions (right medial frontal gyrus, precentral gyrus, and inferior parietal lobe). While these results may seem inconsistent, both higher and lower activation in different brain regions have been reported in order to maintain cognitive performance in studies of aging and various diseases $(26,27,28)$. Differences in activation (in either direction) with the same test performance can be interpreted as the need

Table 2 Comparison of neural activation by fMRI between PHPT and healthy controls.

\begin{tabular}{|c|c|c|c|c|c|c|}
\hline \multirow[b]{2}{*}{ Task } & \multirow{2}{*}{$\begin{array}{l}\text { Activation PHPT } \\
\text { vs controls }\end{array}$} & \multirow[b]{2}{*}{ Voxel number (k) } & \multicolumn{3}{|c|}{ MNI brain coordinates } & \multirow[b]{2}{*}{ Brain region } \\
\hline & & & $\mathrm{x}$ & $\mathrm{Y}$ & $\mathrm{Z}$ & \\
\hline \multirow[t]{4}{*}{ Matrix Reasoning } & Lower & 90 & 9 & -54 & -30 & Left Brainstem \\
\hline & Lower & 72 & 3 & 15 & 60 & $\begin{array}{l}\text { Right Cerebrum; Frontal Lobe; Medial Frontal } \\
\text { Gyrus }\end{array}$ \\
\hline & Lower & 57 & -30 & -18 & 48 & Left Cerebrum; Frontal Lobe \\
\hline & Lower & 51 & 45 & -9 & 60 & Right Cerebrum; Frontal Lobe; Precentral Gyrus \\
\hline Paired Associates & Higher & 65 & 57 & -24 & 27 & $\begin{array}{l}\text { Right Cerebrum; Parietal Lobe; Inferior Parietal } \\
\text { Lobule }\end{array}$ \\
\hline
\end{tabular}


Table 3 Correlation between PHPT biochemistries and task-related activation.

\begin{tabular}{|c|c|c|}
\hline Task & Direction of association & Voxel number $(\mathrm{k})$ \\
\hline \multicolumn{3}{|l|}{ Calcium } \\
\hline Matrix Reasoning & Positive & 69 \\
\hline Paired Associates & Positive & 58 \\
\hline \multicolumn{3}{|l|}{ PTH } \\
\hline \multirow[t]{2}{*}{ Matrix Reasoning } & Positive & 55 \\
\hline & Negative & 64 \\
\hline Paired Associates & Positive & 96 \\
\hline Logical Memory & Positive & 54 \\
\hline
\end{tabular}

\begin{tabular}{|c|c|c|}
\hline \multicolumn{3}{|c|}{ MNI brain coordinates } \\
\hline $\mathrm{X}$ & $Y$ & $\mathrm{Z}$ \\
\hline-42 & -6 & -39 \\
\hline-57 & -36 & 0 \\
\hline-9 & 63 & 27 \\
\hline-9 & 30 & -12 \\
\hline 51 & -48 & 45 \\
\hline-33 & 54 & 18 \\
\hline
\end{tabular}

Brain region

Left middle temporal gyrus Left middle temporal gyrus

Left superior frontal gyrus

Left caudate head

Right inferior parietal lobule Left middle frontal gyrus

MNI, Montreal Neurological Institute.

for recruitment of compensatory brain activity, regions, networks, or mechanisms to maintain equal cognitive performance, consistent with a detrimental effect of PHPT upon the brain. This may be consonant with feelings of 'mental fogginess' or increased mental work that many patients with PHPT report.

There is a precedent for this in several other conditions, including lupus, multiple sclerosis, schizophrenia, and aging $(27,29,30)$. For example, most studies indicate that patients with neuropsychiatric lupus perform comparably to healthy controls on cognitive tests, but have increased activation in the fronto-parietal lobes, which is thought to be necessary to preserve cognitive function (27). In aging, many studies have suggested that maintained cognitive performance with aging tended to be associated with greater activation in the frontal and parietal lobes and temporal cortex, whereas both greater and lower activation in the occipital cortex and hippocampal/medial temporal cortex were associated with better cognitive aging (26).

To investigate if PHPT severity was associated with neuronal function, we assessed correlations of serum calcium and PTH levels with neural activation. We found that both higher PTH and calcium tended to be associated with greater activation in various parts of the brain, including the inferior parietal lobule where activation was higher in those with PHPT compared to controls during the paired associates task. This may suggest that with greater biochemical severity (reflected by higher PTH and calcium), brain activation must increase to compensate to maintain function. However, we also found correlations in both directions between biochemical indicators of PHPT severity and neural activation in brain regions or during tasks where neural activation did not differ between those with and without PHPT. The significance of these latter findings is not clear.

We cannot further isolate the individual roles of calcium vs. PTH in the pathophysiology with the current analysis given the design and associations with both biochemistries. Both PTH and calcium have been associated with worse cognition in other studies (5, 17, 31, 32, 33). The exact pathways by which PTH and calcium affect cognition have not been fully delineated. Hypercalcemia has been proposed to cause cerebral vasospasm or endothelial dysfunction (31). Calcium also plays a key role in regulating release of neurotransmitters at synaptic junctions. Hypercalcemia could interfere with that process. PTH may also have effects on cerebrovascular function (32). Both may act through other mechanisms. The goal of the current analysis was to assess whether neural activation was associated with disease state, namely PHPT, rather than isolate the effects of PTH vs calcium. Future studies that include those with normocalcemic PHPT could more clearly elucidate the contribution of calcium vs. PTH to differences in neural activation.

Table 4 Biochemical changes after PTX.

Serum calcium $(\mathrm{mg} / \mathrm{dL})$
Serum PTH $(\mathrm{pg} / \mathrm{mL})$
Serum 25 -hydroxyvitamin D $(\mathrm{ng} / \mathrm{mL})$
Phosphate $(\mathrm{mg} / \mathrm{dL})$
Creatinine $(\mathrm{mg} / \mathrm{dL})$

\begin{tabular}{c}
\hline Pre-PTX \\
\hline $10.4 \pm 0.4$ \\
$77.2 \pm 30.5$ \\
$40.2 \pm 18.7$ \\
$3.3 \pm 0.5$ \\
$0.77 \pm 0.12$ \\
\hline
\end{tabular}

\begin{tabular}{c}
\hline Post-PTX \\
\hline $9.4 \pm 0.4$ \\
$43.4 \pm 19.2$ \\
$46.9 \pm 19.1$ \\
$3.7 \pm 0.5$ \\
$0.79 \pm 0.13$ \\
\hline
\end{tabular}

\begin{tabular}{c}
\hline P-value \\
\hline$<0.0001$ \\
0.0002 \\
0.004 \\
0.03 \\
0.63
\end{tabular}

PTH, parathyroid hormone; PTX, parathyroidectomy. 


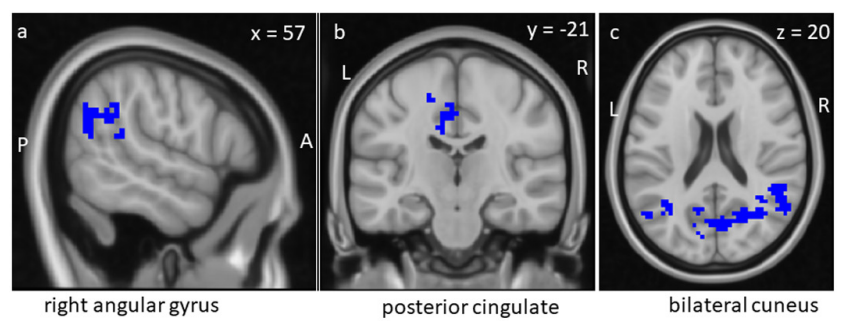

and precuneus

\section{Figure 2}

Representative fMRI images showing the decrease (blue) in neural activation during matrix reasoning between baseline and post-PTX in the right angular gyrus (panel A), posterior cingulate (panel B) and bilateral cuneus, and precuneus (panel $C)$. $X$ refers to the $\mathrm{MNI}$ coordinates in the left-right plane; $Y$ refers to the $\mathrm{MNI}$ coordinates in the posterior-anterior plane; $Z$ refers to the coordinates in the inferior-superior plane.

The observed different patterns of neural activation were not 'reversible' with cure of PHPT after PTX. This could indicate that PHPT has a permanent effect on processing or more time is required to allow for improvement. Further work is needed to fully assess the implications of this finding. There is a parallel for this in terms of the skeletal changes that occur after PTX in PHT. While PHPT is clearly recognized to cause preferential loss of bone mineral density (BMD) at the one-third radius, BMD at this skeletal site does not improve after PTX or only does so after many years (1). The current study followed PHPT patients for 6 months after surgery. This duration was selected based on our prior study indicating improvement in verbal memory and non-verbal abstraction at 6 months post-PTX.

While the absence of inferior task performance or post-PTX improvement in neural activation does not support changing surgical guidelines for PHPT to include cognitive symptoms, the small number of participants followed longitudinally limits the overall conclusions that can be drawn regarding the effect of PTX upon these parameters. Further, we cannot determine if the patterns of neural activation would have worsened without PTX or as a function of time due to the absence of a non-operated PHPT comparator group. The post-PTX changes in neural activation observed in other brain regions, not affected pre-operatively, could be related to learning. Future studies will require a longitudinal control group to better elucidate the meaning of these post-operative changes in neural activation.

Only two studies have utilized fMRI to study cognition in PHPT and both lacked a comparison to normal controls. The first was a feasibility study of six patients assessed with fMRI before and 3 weeks after PTX (21). The authors reported an increase in activation in the medial prefrontal cortex and parietal lobe after surgery during an executive function task (the Stroop task) and a decrease in the dorsolateral prefrontal cortex (21). The same group conducted a small, randomized controlled trial of 18 patients with PHPT assessing the effect of PTX or observation upon neural activation during the Stroop task (13). There were no differences in neural activation over time between groups and no association between change in activation and changes in serum PTH or calcium (13). Inconsistencies between these studies and ours may be due to differences in the design, tasks utilized, fMRI techniques, and the size of the studies, among other factors.

In contrast to our prior study assessing cognition using traditional cognitive testing without fMRI (5), we did not find that verbal memory or executive function test/task performance was worse in those with PHPT compared to controls. The reasons for this are unclear. The specific tests utilized differed between our studies, but targeted the same cognitive demands (verbal memory and fluid reasoning). In the current study, the groups were well-matched to controls in age, education, and comorbidities and we excluded conditions known to affect cognition other than PHPT. Studies regarding the presence of cognitive symptoms in PHPT and improvement post-PTX have had inconsistent results $(5,6,7,8,9,10,11,12,13,14,15,16$, 17). A recent systemic review that summarized the results of 13 observational pre-post PTX studies concluded there was mixed evidence for improvement in memory after PTX and very limited agreement in other cognitive domains (17). It is possible that biochemical severity accounts for some differences in cognitive task performance between our study and others. Our patients had biochemically very mild PHPT, which is the typical presentation of PHPT in the US today, in contrast to other investigations that included more severe hyperparathyroidism.

As no other studies have compared PHPT to controls and the neural activation patterns in our study were not reversible with PTX, replication of our findings is needed to definitively conclude that the differences in neuronal activity represent a 'disease-specific' pattern. To this end, however, we used well-matched controls from a referent normative fMRI database that has established standard patterns of activation for the given tasks across the lifespan (22). We believe these results are important because, while many patients with PHPT report cognitive symptoms, prior studies have not identified a consistent 
measure to identify them. This and the lack of a clear mechanism have rendered this issue challenging to study and manage clinically. Thus, the presence and reversibility of neuropsychological symptoms have remained the most debated issue in the management of PHPT.

This study provides information regarding tools and testing that may be useful in future PHPT studies $(2,34)$. If other studies confirm a similar 'fMRI fingerprint', then it may offer a useful biomarker to detect subtle effects of PHPT on brain function that have not been objectively and rigorously identified with traditional cognitive testing. Ultimately, such a biomarker could also be valuable in making clinical decisions for patients with PHPT. Functional MRI is appealing because it is safe, non-invasive, and does not involve radiation or contrast. Further, beyond traditional testing, it can identify the brain regions affected and can determine whether there is neural compensation even in the presence of similar cognitive performance.

Our study has several limitations. While our study is large in comparison to other PHPT studies using fMRI, it is small compared to studies assessing cognitive aging or neurodegenerative disorders. However, with the current sample size, we had $>99 \%$ power to detect the effect size for matrix reasoning between cases and controls with a two-tailed alpha of $1 \%$. Since we lacked a longitudinal control group, we cannot determine if the change in neural activation and reaction time post-PTX is due to learning nor can we determine if neural activation may have worsened without PTX. Further, the size of the longitudinal cohort limits conclusions regarding the effect of PTX on neural activation. Additionally, this study was performed in a tertiary care center, participants had very mild PHPT, and many declined participation or were not eligible. We may have found more marked differences in those with biochemically severe PHPT. Our results may not be generalizable to those with severe PHPT and those included may not be representative of all patients with PHPT. Unfortunately, we did not collect data on subjective neuropsychological symptoms and cannot correlate these to biochemistries or neural activation. Lastly, we excluded participants with dementia on the basis of history, but did not formally screen or match cases and controls using a validated dementia instrument such as the mini-mental state exam or another test. The latter approach may have been a more robust method.

Our study also has several strengths. This is the only study to compare neural activation patterns in patients with PHPT compared to healthy controls. Further, we used controls from a normative database that established standard fMRI activation patterns for the given tasks across the lifespan. The study excluded conditions known to affect cognitive performance other than PHPT and our participants were well-matched to those without PHPT with regard to age, education, lifestyle factors, medications, and comorbidities, limiting the effects of these potential cofounders on our analysis. We also assessed the cognitive domains previously shown to be affected in PHPT with validated tests. Additionally, we studied patients well after the immediate postoperative period to prevent biases due to the nonspecific benefits of surgery.

In summary, PHPT is associated with differences in cognitive task-related neural activation by fMRI in brain regions related to executive function and verbal memory. These aspects of cognition include those reported to be affected in prior cognitive studies in PHPT. These altered neural activation patterns were associated with similar task accuracy, which may indicate that there are compensatory mechanisms to maintain cognitive functioning in PHPT. Future larger, longitudinal studies that include PHPT controls followed without surgery are needed to determine if PTX prevents worsening of altered neural activation patterns in PHPT.

\section{Declaration of interest}

The authors declare that there is no conflict of interest that could be perceived as prejudicing the impartiality of this study.

\section{Funding}

NIH R21 DK104105, UL1TR000040, R01AG026158, K01AG051777, RFAG038465, Endocrine Fellows Foundation, and Columbia University Aging Center Grant.

\section{References}

1 Silverberg SJ, Shane E, Jacobs TP, Siris E \& Bilezikian JP. A 10-year prospective study of primary hyperparathyroidism with or without parathyroid surgery. New England Journal of Medicine 1999341 1249-1255. (https://doi.org/10.1056/NEJM199910213411701)

2 Walker MD \& Silverberg SJ. Primary hyperparathyroidism. Nature Reviews: Endocrinology 201814 115-125. (https://doi.org/10.1038/ nrendo.2017.104)

3 Walker MD \& Bilezikian JP. Primary hyperparathyroidism: recent advances. Current Opinion in Rheumatology 201830 427-439. (https:// doi.org/10.1097/BOR.0000000000000511)

4 Chiodini I, Cairoli E, Palmieri S, Pepe J \& Walker MD. Non classical complications of primary hyperparathyroidism. Best Practice and Research: Clinical Endocrinology and Metabolism 201832 805-820. (https://doi.org/10.1016/j.beem.2018.06.006)

5 Walker MD, McMahon DJ, Inabnet WB, Lazar RM, Brown I, Vardy S, Cosman F \& Silverberg SJ. Neuropsychological features in primary hyperparathyroidism: a prospective study. Journal of Clinical Endocrinology and Metabolism 200994 1951-1958. (https://doi. org/10.1210/jc.2008-2574) 
6 Sheldon DG, Lee FT, Neil NJ \& Ryan Jr JA. Surgical treatment of hyperparathyroidism improves health-related quality of life. Archives of Surgery 2002137 1022-1026; discussion 1026-1028. (https://doi. org/10.1001/archsurg.137.9.1022)

7 Amstrup AK, Rejnmark L \& Mosekilde L. Patients with surgically cured primary hyperparathyroidism have a reduced quality of life compared with population-based healthy sex-, age-, and seasonmatched controls. European Journal of Endocrinology 2011165 753-760. (https://doi.org/10.1530/EJE-11-0301)

8 Bollerslev J, Jansson S, Mollerup CL, Nordenstrom J, Lundgren E, Torring O, Varhaug JE, Baranowski M, Aanderud S, Franco C et al. Medical observation, compared with parathyroidectomy, for asymptomatic primary hyperparathyroidism: a prospective, randomized trial. Journal of Clinical Endocrinology and Metabolism 200792 1687-1692. (https://doi.org/10.1210/jc.2006-1836)

9 Ambrogini E, Cetani F, Cianferotti L, Vignali E, Banti C, Viccica G, Oppo A, Miccoli P, Berti P, Bilezikian JP et al. Surgery or surveillance for mild asymptomatic primary hyperparathyroidism: a prospective, randomized clinical trial. Journal of Clinical Endocrinology and Metabolism 200792 3114-3121. (https://doi.org/10.1210/jc.2007-0219)

10 Talpos GB, Bone 3rd HG, Kleerekoper M, Phillips ER, Alam M, Honasoge M, Divine GW \& Rao DS. Randomized trial of parathyroidectomy in mild asymptomatic primary hyperparathyroidism: patient description and effects on the SF-36 health survey. Surgery 2000128 1013-1020; discussion 1020-1011. (https://doi.org/10.1067/msy.2000.110844)

11 Roman SA, Sosa JA, Mayes L, Desmond E, Boudourakis L, Lin R, Snyder PJ, Holt E \& Udelsman R. Parathyroidectomy improves neurocognitive deficits in patients with primary hyperparathyroidism. Surgery 2005138 1121-1128; discussion 1128-1129. (https://doi.org/10.1016/j.surg.2005.08.033)

12 Benge JF, Perrier ND, Massman PJ, Meyers CA, Kayl AE \& Wefel JS. Cognitive and affective sequelae of primary hyperparathyroidism and early response to parathyroidectomy. Journal of the International Neuropsychological Society 200915 1002-1011. (https://doi. org/10.1017/S1355617709990695)

13 Perrier ND, Balachandran D, Wefel JS, Jimenez C, Busaidy N, Morris GS, Dong W, Jackson E, Weaver S, Gantela S et al. Prospective, randomized, controlled trial of parathyroidectomy versus observation in patients with 'asymptomatic' primary hyperparathyroidism. Surgery 2009146 1116-1122. (https://doi. org/10.1016/j.surg.2009.09.034)

14 Chiang CY, Andrewes DG, Anderson D, Devere M, Schweitzer I \& Zajac JD. A controlled, prospective study of neuropsychological outcomes post parathyroidectomy in primary hyperparathyroid patients. Clinical Endocrinology 200562 99-104. (https://doi. org/10.1111/j.1365-2265.2004.02180.x)

15 Numann PJ, Torppa AJ \& Blumetti AE. Neuropsychologic deficits associated with primary hyperparathyroidism. Surgery 198496 1119-1123.

16 Babinska D, Barczynski M, Stefaniak T, Oseka T, Babinska A, Babinski D, Sworczak K, Lachinski AJ, Nowak W \& Sledzinski Z. Evaluation of selected cognitive functions before and after surgery for primary hyperparathyroidism. Langenbeck's Archives of Surgery 2012397 825-831. (https://doi.org/10.1007/s00423-011-0885-5)

17 Lourida I, Thompson-Coon J, Dickens CM, Soni M, Kuzma E, Kos K \& Llewellyn DJ. Parathyroid hormone, cognitive function and dementia: a systematic review. PLoS ONE 201510 e0127574. (https:// doi.org/10.1371/journal.pone.0127574)

18 Walker MD \& Silverberg SJ. Parathyroidectomy in asymptomatic primary hyperparathyroidism: improves 'bones' but not 'psychic moans'. Journal of Clinical Endocrinology and Metabolism 200792 1613-1615. (https://doi.org/10.1210/jc.2007-0551)

19 Habeck C, Eich T, Razlighi R, Gazes Y \& Stern Y. Reference ability neural networks and behavioral performance across the adult life span. Neuroimage 2018172 51-63. (https://doi.org/10.1016/j. neuroimage.2018.01.031)

20 Finkelmeyer A, Nilsson J, He J, Stevens L, Maller JJ, Moss RA, Small S, Gallagher P, Coventry K, Ferrier IN et al. Altered hippocampal function in major depression despite intact structure and resting perfusion. Psychological Medicine 201646 2157-2168. (https://doi. org/10.1017/S0033291716000702)

21 Perrier ND, Coker LH, Rorie KD, Burbank NS, Kirkland KA, Passmore LV, Tembreull T, Stump DA \& Laurienti PJ. Preliminary report: functional MRI of the brain may be the ideal tool for evaluating neuropsychologic and sleep complaints of patients with primary hyperparathyroidism. World Journal of Surgery $2006 \mathbf{3 0}$ 686-696. (https://doi.org/10.1007/s00268-005-0361-x)

22 Stern Y, Habeck C, Steffener J, Barulli D, Gazes Y, Razlighi Q, Shaked D \& Salthouse T. The Reference Ability Neural Network Study: motivation, design, and initial feasibility analyses. Neuroimage 2014103 139-151. (https://doi.org/10.1016/j. neuroimage.2014.09.029)

23 Habeck C, Gazes Y, Razlighi Q, Steffener J, Brickman A, Barulli D, Salthouse T \& Stern Y. The Reference Ability Neural Network Study: life-time stability of reference-ability neural networks derived from task maps of young adults. Neuroimage 2016125 693-704. (https:// doi.org/10.1016/j.neuroimage.2015.10.077)

24 Walker MD, Cong E, Kepley A, DiTullio MR, Rundek T, Homma S, Lee JA, Liu R, Young P, Zhang C et al. Association between serum 25-hydroxyvitamin D level and subclinical cardiovascular disease in primary hyperparathyroidism. Journal of Clinical Endocrinology and Metabolism 201499 671-680. (https://doi.org/10.1210/jc.2013-3523)

25 Bilezikian JP, Brandi ML, Eastell R, Silverberg SJ, Udelsman R, Marcocci C \& Potts Jr JT. Guidelines for the management of asymptomatic primary hyperparathyroidism: summary statement from the Fourth International Workshop. Journal of Clinical Endocrinology and Metabolism 201499 3561-3569. (https://doi. org/10.1210/jc.2014-1413)

26 Eyler LT, Sherzai A, Kaup AR \& Jeste DV. A review of functional brain imaging correlates of successful cognitive aging. Biological Psychiatry 201170 115-122. (https://doi.org/10.1016/j.biopsych.2010.12.032)

27 Barraclough M, Elliott R, McKie S, Parker B \& Bruce IN. Cognitive dysfunction and functional magnetic resonance imaging in systemic lupus erythematosus. Lupus 201524 1239-1247. (https://doi. org/10.1177/0961203315593819)

28 Barraclough M, McKie S, Parker B, Jackson A, Pemberton P, Elliott R \& Bruce IN. Altered cognitive function in systemic lupus erythematosus and associations with inflammation and functional and structural brain changes. Annals of the Rheumatic Diseases 2019 78 934-940. (https://doi.org/10.1136/annrheumdis-2018-214677)

29 Mikdashi JA. Altered functional neuronal activity in neuropsychiatric lupus: a systematic review of the fMRI investigations. Seminars in Arthritis and Rheumatism 201645 455-462. (https://doi. org/10.1016/j.semarthrit.2015.08.002)

30 Wager TD \& Smith EE. Neuroimaging studies of working memory: a meta-analysis. Cognitive, Affective and Behavioral Neuroscience 20033 255-274. (https://doi.org/10.3758/cabn.3.4.255)

31 Camara-Lemarroy CR, Gonzalez-Moreno EI, Ortiz-Corona Jde J, Yeverino-Castro SG, Sanchez-Cardenas M, Nuñez-Aguirre S, Villarreal-Alarcon MA \& Galarza-Delgado DA. Posterior reversible encephalopathy syndrome due to malignant hypercalcemia: physiopathological considerations. Journal of Clinical Endocrinology and Metabolism 201499 1112-1116. (https://doi.org/10.1210/ jc.2013-3487)

32 Liu M, Sum M, Cong E, Colon I, Bucovsky M, Williams J, Kepley A, Kuo J, Lee JA, Lazar RM et al. Cognition and cerebrovascular function in primary hyperparathyroidism before and after parathyroidectomy. Journal of Endocrinological Investigation 202043 369-379. (https://doi. org/10.1007/s40618-019-01128-0) 
33 Roman SA, Sosa JA, Pietrzak RH, Snyder PJ, Thomas DC, Udelsman R $\&$ Mayes L. The effects of serum calcium and parathyroid hormone changes on psychological and cognitive function in patients undergoing parathyroidectomy for primary hyperparathyroidism. Annals of Surgery 2011253 131-137. (https://doi.org/10.1097/ SLA.0b013e3181f66720)
34 Silverberg SJ, Clarke BL, Peacock M, Bandeira F, Boutroy S, Cusano NE, Dempster D, Lewiecki EM, Liu JM, Minisola S et al. Current issues in the presentation of asymptomatic primary hyperparathyroidism: proceedings of the Fourth International Workshop. Journal of Clinical Endocrinology and Metabolism 2014 99 3580-3594. (https://doi.org/10.1210/jc.2014-1415)

Received 14 February 2020

Revised version received 15 April 2020

Accepted 29 April 2020 